\title{
A humanização do cuidado ao recém-nascido portador de hidrocefalia e seus cuidadores: a contribuição da Enfermagem
}

\author{
Cláudio José de Souza, M.Sc.*, Marcela Cristina Lemos Heringer**, Geilsa Soraia Cavalcanti Valente, D.Sc.***
}

${ }^{*}$ Enfermeiro, Docente e Coordenador Adjunto do Curso de Pós-Graduação em Terapia Intensiva pela Faculdade Bezerra de Araújo, **Enfermeira Pós-Graduanda em Terapia Intensiva pela Faculdade Bezerra de Araújo, Rio de Janeiro RJ, ***Professora Adjunta da Escola de Enfermagem Aurora de Afonso Costa da Universidade Federal Fluminensel UFF

\section{Resumo}

Objetivo: Identificar e discutir, através de revisão sistematizada da literatura, as contribuiçôes de enfermagem visando à assistência humanizada ao recém-nascido $(\mathrm{RN})$ portador de hidrocefalia e aos seus familiares. Método: Revisão bibliográfica do tipo exploratória, com abordagem qualitativa. A pesquisa foi realizada na Biblioteca Virtual de Saúde (BVS), nas bases Lilacs, Scielo, BDENF e Medline. Após a coleta de dados, realizou-se a leitura exploratória, seletiva, analítica e interpretativa. Resultados: Os estudos evidenciaram que o diagnóstico precoce pode ser obtido através de exames específicos e que ainda há uma série de questóes que podem potencializar a doença trazendo maiores riscos de complicaçóes e óbitos mediante as intervenções realizadas. Conclusão: A enfermagem tem papel preponderante na atuação não só ao RN portador de hidrocefalia, mas também a seus familiares. Destacando que este pode ser um diferencial, voltado às bases da humanização, do cuidado individualizado e de excelência.

Palavras-chave: Unidades de Terapia Intensiva Neonatal, hidrocefalia, cuidados de enfermagem, parto humanizado.

\section{Abstract}

Humanization of care of the newborn with hydrocephalus and their caregivers: the contribution of Nursing

Objective: To identify and discuss, through a systematic literature review, nursing contributions aiming at humanized care to newborn with hydrocephalus and their family. Methods: This is a literature review of exploratory and qualitative approach. The research was conducted in the Virtual Health Library (VHL), at Lilacs, Scielo, Medline and BDENF databases. After data collection, an exploratory, selective, analytical and interpretive reading was carried out. Results: The study showed that specific test may provide early diagnosis and that there are still a number of issues that may intensify the disease carrying higher risks of complications and deaths during interventions. Conclusion: The nursing has an important 
role of supporting not only the newborn with hydrocephalus, but also their families. We can highlight that this may be a differential based on humanization, providing individualized care with excellence.

Key-words: Intensive Care Units, neonatal, hydrocephalus, nursing care, humanizing delivery.

\section{Resumen}

\section{Humanización de la atención al recién nacido portador de hidrocefalia y sus cuidadores: la contribución de enfermería}

Objetivo: Identificar y analizar, a través de una revisión sistemática de la literatura, las contribuciones de enfermería relacionadas a la asistencia humanizada del recién nacido portador hidrocefalia y de sus familias. Métodos: Revisión de la literatura de enfoque exploratorio, cualitativo. La investigación se realizó en la Biblioteca Virtual en Salud (BVS), en las bases de datos Lilacs, Scielo, Medline y BDENF. Después de la recogida de datos, se llevó a cabo la lectura exploratoria, selectiva, analítica e interpretativa. Resultados: El estudio mostró que el diagnóstico precoz se puede obtener por medio de pruebas específicas y que todavía hay una serie de cuestiones que pueden potencializar la enfermedad y traer mayores riesgos de complicaciones y muertes debido a las intervenciones. Conclusión: El equipo de enfermería tiene el papel preponderante en el soporte no sólo al recién nacido con hidrocefalia, sino también a sus familias. Se destaca que este puede ser un diferencial, basado en los fundamentos de la humanización, de la atención personalizada y de excelencia.

Palabras-clave: Unidades de Cuidado Intensivo Neonatal, atención de enfermería, parto humanizado.

\section{Introdução}

A essência da arte da enfermagem reside no cuidado que transcende o indivíduo enfermo e se estende a todos aqueles que sofrem influência direta ou indiretamente. Enquanto profissionais preocupados com a humanização do cuidado, devemos valorizar as relações com os assistidos e os pares. Tendo em mente que esta apreensão da humanização não deve estar voltada somente para o cuidado do paciente em si, mas também para os seus familiares e entes queridos. Entender que quando se presta o cuidado devemos fazê-lo sem distinção e sem prioridades, mas sim fazê-lo de forma integral e igualitária a todos [1].

É imprescindível que a assistência de enfermagem seja qualificada e entendida como direito de todos, incluindo os assistentes e os assistidos, abarcando, não só a necessidade e a garantia dessa assistência, mas também fomentar os princípios que regem a profissão pautada na ética social, universalidade, acesso uquânime, segurança, responsabilidade, atendimento de qualidade, solidariedade. Esses são entendidos como elementos fundamentais à expressão do direito à saúde de qualquer cidadão [2]

A hidrocefalia é entidade nosológica definida como aumento da quantidade de líquido cefalorraquidiano ou líquor nas cavidades ventriculares e espaço subaracnóideo, podendo acometer o espaço subdural. Sua principal decorrência clínica imediata é a hipertensão intracraniana, necessitando de atendimento imediato de cunho cirúrgico [3].

O termo hidrocefalia é derivado de duas palavras gregas, na qual "hidro" significa água e "cefálico" é igual à cabeça. A hidrocefalia tem como principal característica o excesso de líquido denominado líquor cefalorraquidiano na calota cerebral. Esse excesso causa dilatação dos ventrículos aumentando assim a pressão nociva dos tecidos cerebrais.

O motivo do aumento da produção excessiva desse líquido cefalorraquiadiano pode ser devido a uma má absorção ou obstrução que impede o seu fluxo. O líquido cefalorraquidiano tem seu volume total em torno de 125 a $150 \mathrm{ml}$, sua pressão normal em repouso é de 150 a $180 \mathrm{mmH}_{2} \mathrm{O}$ e sua produção é de 0,2 a $0,7 \mathrm{ml} / \mathrm{min}$. Normalmente, flui dos ventrículos laterais através do forame de Monroe para o terceiro ventrículo, percorre o estreito do arqueduto de Sylvius e entra no quarto ventrículo. Deixa o quarto ventrículo através do par de forames de Luschka e forame de Magendie e ganha às cisternas da base do encéfalo [4].

A hidrocefalia é uma categoria em que os ventrículos do cérebro estão acrescidos em detrimento do desequilíbrio entre a produção e a absorção do líquido cefalorraquidiano. É quase sempre ocasionada pela interferência com a circulação e com a absorção do líquor. A hidrocefalia congênita comumente apa- 
rece em razão de uma malformação no cérebro ou de uma infecçâo intrauterina, esta frequentemente aparece em conjunto com a meningomielocele, que bloqueia o fluxo do líquor [5].

Qualquer desequilíbrio da secreção e absorção causa um aumento do acúmulo de LCR nos ventrículos, os que se tornam dilatados e comprimem a substância encefálica contra o crânio ósseo rígido circundante. As causas da hidrocefalia no recém-nascido incluem neoplasias, infecçóes, má formação congênita, hemorragia intracraniana e traumatismo. Pode haver uma obstrução em qualquer ponto do trajeto do líquido para produzir aumento da pressão e dilatação das vias ao local de produção, malformação de Arnold-Chiari que consiste na herniação do tronco cerebral e cerebelo inferior através do forame magno para dentro do canal vertebral cervical [6].

Dentre as causas e fatores predisponentes da hidrocefalia destacam-se: causas congênitas que incluem estenose de aqueduto de Syvius; hereditariedade ligada ao cromossomo $\mathrm{X}$; defeito do tubo neural com malformação; lesôes císticas congênitas do mecanismo obstrutivo, como: cisto aracnóide, ependimário, por encefálico e linha mediana e tumores congênitos do mecanismo obstrutivo. As infecções intrauterinas, em geral, podem levar a malformação anatômica bem como meningites que podem causar bloqueio do fluxo de LCR, como no caso de toxoplasmose, vírus de inclusão citomegálica, varíola, estafilococos e sífilis. $\mathrm{E}$, por fim, a obstrução pós-hemorrágica que inclui o aneurisma da veia de Galeno. Suas principais causas são hereditariedade, má-formação do desenvolvimento cerebral, cisto benigno, tumores congênitos, infecção intracraniana, traumatismo, injeção intrauterina, hemorragia perinatal [7].

A hidrocefalia refere-se à dilatação anormal dos ventrículos laterais. Podem ser classificados como comunicante ou não comunicante; o comunicante a obstrução do fluxo é extraventricular, ao nível do espaço subaracnóideo; já a não comunicante caracteriza-se pela obstrução do fluxo cefalorraquidiano no sistema intraventricular [8].

Caracteriza-se como hidrocefalia comunicante, podendo também ser chamada de externa, a que causa obstrução fora do sistema ventricular, aumentando, assim, a dimensão dos ventrículos, mas continuando a comunicação entre os líquidos ventriculares e os subaracnóideos. A hidrocefalia não-comunicante, também conhecida como interna, obstrui dentro do sistema ventricular, não fazendo comunicação com os líquidos aracnóides [9].

Devido à grande seriedade da patologia em questáo, fica notória a necessidade de o enfermeiro possuir um conhecimento técnico e científico acerca do assunto, uma vez que os sinais clínicos e dados técnicos necessitam ser explanados com o intuito de produzir informaçóes sobre o real estado da doença atual desse paciente.

O enfermeiro analisa os comportamentos ou respostas dos clientes que refletem uma regressão ou melhoria no diagnóstico de enfermagem ou a conservação de um estado saudável. Durante a evolução, o enfermeiro decide se as etapas anteriores do processo de enfermagem foram eficazes, examinando as respostas dos clientes e comparando-as aos comportamentos estabelecidos e aos resultados esperados [10].

Desta forma, consideram que a cientificação profissional é almejada pelos profissionais de enfermagem e só será alcançada com a utilização de instrumentos científicos que subsidiem a prática profissional [11].

O objetivo deste estudo foi identificar e discutir as contribuições de enfermagem visando à assistência humanizada ao recém-nascido $(\mathrm{RN})$ portador de hidrocefalia e aos seus familiares.

\section{Material e métodos}

Tratou-se de uma pesquisa qualitativa, bibliográfica, do tipo exploratória, pois tem como finalidade proporcionar maior familiaridade com o problema, com vistas a torná-lo mais explícito [12]. Empregou estudos primários identificados na biblioteca Virtual de Saúde (BVS), visando atender à recomendação da literatura de que se busquem diferentes fontes de dados.

Foi realizado um levantamento bibliográfico, nas respectivas bases de dados, utilizando os descritores: unidades de terapia intensiva, hidrocefalia, cuidados de enfermagem e parto humanizado num primeiro momento individual. Obtendo os seguintes resultados: Unidades de Terapia Intensiva Neonatal: Scielo (52) Medline (8.511) Lilacs (547) BDENF (1570); Hidrocefalia Scielo (206) Medline (15.995) Lilacs (333) BDENF (1); Cuidados de Enfermagem Scielo (517) Medline (25.148) Lilacs (3.292) BDENF (2.838); Parto Humanizado Scielo (28) Medline (0) Lilacs (181) BDENF (110). 
Após a coleta de dados, realizou-se uma leitura exploratória, a qual se caracteriza por ser uma leitura corrida, objetivando evidenciar se o texto atendia ao objetivo proposto. Depois, realizou-se uma leitura seletiva, elegendo e avaliando a contribuição de cada texto e seus principais tópicos eliminando os repetidos [13].

\section{Resultados e discussão}

A pesquisa abarcou a bibliografia potencial de 9 artigos, sendo 6 da base Scielo; 2 da base Lilacs 1 da base BDENF, os quais evidenciaram desde os métodos diagnóstico intrauterino para hidrocefalia perpassando pelas complicaçóes e as intervençóes cirúrgicas que a patologia em voga pode necessitar como terapêutica, transitando na área do conhecimento dos familiares e finalizando com a assistência de enfermagem com abordagem holística.

Em estudo que utilizou os testes de $\mathrm{C}^{2}$ e exato de Fisher, com base na distribuição e média do público pesquisado, o objetivo foi avaliar os métodos para diagnóstico, características da gestação, complicaçóes maternas e resultados perinatais em casos de hidrocefalia congênita, correlacionando-os com variáveis gestacionais e do parto. Evidenciou-se que o diagnóstico de hidrocefalia foi anterior ao parto, confirmado pelo exame ecográfico, e o parto foi por cesárea na maioria dos casos. A cefalocentese foi realizada em 11 casos e as complicaçóes no parto vaginal foram mais frequentes que na cesárea. $\mathrm{O}$ índice de Apgar baixo foi mais frequente entre os recém-nascidos de parto vaginal. Pode ser constatado que a avaliaçáo destes fatores pode ser um diferencial no acompanhamento das gestantes com esta malformação fetal, dando maior respaldo às decisões que, além de médicas e éticas, devem levar em conta a relação risco-benefício das medidas a serem tomadas [14].

Através de estudo retrospectivo com 243 casos de hidrocefalia com início na infância, analisaram-se as características epidemiológicas e clínicas com hidrocefalia derivada na faixa etária de 1 a 27 anos e identificaram-se os fatores clínicos relacionados às complicaçóes mais frequentes, tendo como destaque distúrbios psicomotores, epilepsia e o óbito. Constatou-se que a meningite pós-derivação e os distúrbios mecânicos do sistema foram as complicações mais frequentes. Os distúrbios motores correlacionaram-se positivamente com o grau da hidrocefalia. Houve maior incidência de déficit cognitivo e epilepsia nos casos de hidrocefalia pós-meningite. $\mathrm{O}$ óbito ocorreu em 52 pacientes e estes apresentaram um percentual maior de neoplasia do sistema nervoso central e de distúrbios motores na última avaliação, assim como de retirada da primeira derivaçáo por meningite precoce, ocorrida até dois meses após a derivação [15].

Estudo descritivo, em pacientes pós-cirúrgicos de hidrocefalia ventrículo peritoneal, determinou as principais complicaçóes pós-operatórias. Os resultados evidenciaram que os altos índices de complicaçóes estavam relacionados à disfunção do sistema, infecção e hematoma devido à drenagem, o que constatou que a hidrocefalia congênita apresenta um maior índice de complicaçóes pós-cirúrgica [16].

Estudo prospectivo, observacional náo controlado, realizado com 50 pacientes que foram submetidos à derivaçáo ventricular peritoneal, determinou a frequência e as causas de infecção pós-implantação dos dispositivos. Destes foram destacados que a maior parte das infecçóes está relacionada à infecção do sítio cirúrgico, mostrando a necessidade de um olhar diferenciado aos profissionais que atuam diretamente nos cuidados do cateter, pois foi demonstrado pelo estudo que náo houve alteraçóes significativas quanto à idade, à etiologia da hidrocefalia, tipo de procedimento, tempo de internação pré-operatória, duração da cirurgia, antibiótico-profilaxia, cateter SNC prévio e índice de risco cirúrgico [17].

No estudo descritivo-exploratório realizado com 54 cuidadores de crianças com hidrocefalia congênita, o objetivo foi identificar fatores socioeconômico, social e cultural destes cuidadores bem como seus conhecimentos sobre a patologia em voga. Foi identificado que as maiores parcelas destes cuidadores são do sexo feminino e que poucas obtiveram informaçóes de como proceder no cuidado ao paciente. Além disso, o fator cultural (escolaridade) é importante para maior conhecimento da patologia [18].

Trabalho de conclusão de curso, com abordagem qualitativa, por meio de entrevistas semiestruturadas e categorizaçáo dos dados, teve como objetivo conhecer o impacto da primeira visita à criança internada na Unidade de Tratamento Intensivo Neonatal para sua máe. Foram destacadas categorias importantes no que tange a percepção destes familiares nesta unidade e foram pontuados os seguintes itens: a unidade é reconhecida como um setor para o atendimento de pacientes graves; a 
tecnologia existente é causadora de estresse; o entrar desacompanhada e receber poucas informaçóes acerca da criança podem dificultar a presença da mãe na unidade e tornar a visita assustadora gerando sentimentos contraditórios. Evidenciou-se a necessidade do preparo destes familiares, com fornecimento de informaçóes simples e relevantes capazes de diminuir a angústia e o temor, tendo como ponto crucial o apoio da equipe de enfermagem junto a esta clientela principalmente no momento da visita, fortalecendo seu vínculo afetivo com a criança [19].

Estudo qualitativo de abordagem da fenomenologia social teve com alvo compreender como os enfermeiros vivenciam o cuidado prestado ao recém-nascido, na presença dos pais. Foram destacadas na pesquisa as seguintes categorias: que os enfermeiros percebem as necessidades dos pais; tem expectativas positivas em relação ao cuidado realizado e reconhece ser o elo entre eles, ajudando-os a conviver com o filho internado. Porém um dos fatores dificultadores para a assistência é quando ocorrem situações de emergência em que os profissionais precisam executar os procedimentos frente aos pais. Pelo estudo foi evidenciado que os enfermeiros avaliam positivamente a presença dos pais na unidade de terapia intensiva neonatal, procurando envolvê-los permanentemente no cuidado ao recém-nascido, o que torna o cuidado diferenciado e humanizado para ambos [20].

No estudo exploratório de abordagem qualitativa, o objetivo foi analisar, sob a ótica dos profissionais de saúde, a proposta de atençáo humanizada e detectar os sentidos e os limites identificados por eles para a oferta desta assistência em Unidade de Terapia Intensiva Neonatal. $\mathrm{Na}$ pesquisa foi demonstrado que existem importantes pontos de impedimentos para a oferta da assistência humanizada, como a falta de recursos materiais e humanos, influenciando a sobrecarga de trabalho, conflitos de relacionamento e a falta de infraestrutura, tanto para os trabalhadores como para conduzir as iniciativas de humanizaçáo, como o alojamento de nutrizes. Este estudo revelou que, embora existam obstáculos, os profissionais criam estratégicas para atender ao que foi preconizado na Política Nacional de Humanizaçáo do Ministério da Saúde [21].

$\mathrm{Na}$ dissertação de mestrado realizada do estudo da fenomenologia Alfred Schutz, com abordagem qualitativa, o objetivo do estudo foi aprender os aspectos éticos implícitos nas açóes experienciadas pelos profissionais de enfermagem aos recém-nas- cidos na Unidade de Terapia Intensiva Neonatal. A pesquisa demonstrou que os profissionais que lá atuam procuram realizar o cuidado desejando a cura e a alta do RN, procurando associar a tecnologia com um fator importante na prestaçáo deste cuidado humanizado, procurando prestar a assistência de maneira individualizada e ética. Ressaltando que há uma necessidade de avaliação e reflexão sobre a adequaçáo da tecnologia e a adoção de estratégias que valorizem e respeitem a vida humana em toda sua dimensão, porém que esta abordagem estenda-se não somente aos pacientes, mas sim a seus familiares [22].

Estudo qualitativo, através do referencial metodológico com teoria fundamentada nos dados ou Grounded Theory, buscou compreender o significado do ser e do fazer o cuidado para os enfermeiros em uma Unidade de Tratamento Intensivo Neonatal (UTIN) de um hospital geral do sul do Brasil, construindo um modelo teórico explicativo. A pesquisa demonstrou que o cuidado em UTIN deve valorizar as interrelaçóes cotidianas, além de procurar maior atuação em todas as esferas do cuidado complexo em saúde, agregando e consagrando conhecimentos científicos. Logo, é primordial que se exercite as potencialidades já inatas dos profissionais de enfermagem, por meio de um caminho que busque novas formas de cuidar do neonato e de sua família e os membros deste sistema complexo [23].

Em contrapartida, a pesquisa qualitativa, realizada em um Hospital Universitário de agosto a novembro de 2009, com entrevistas semiestruturadas com dez mães, cujo objetivo foi conhecer o impacto da primeira visita à criança internada na Unidade de Tratamento Intensivo Neonatal para sua mãe, constatou que em um momento inicial é impactante para os pais. A unidade apresenta um estigma de ser um setor para o atendimento de pacientes graves, além de possuir uma tecnologia que causa estresse, devido ao grande número de aparelhos. Logo, o entrar desacompanhada e receber poucas informaçóes sobre a criança pode atrapalhar a presença da mãe na unidade e, desta forma, a visita pode ser assustadora propiciando sentimentos contraditórios. Assim, é imprescindível que a mãe tenha apoio da equipe de enfermagem durante a visita, a fim de fortalecer os vínculos afetivos com a criança [24].

Em um estudo descritivo retrospectivo, no qual foram avaliadas as gestaçóes com diagnóstico de hidrocefalia fetal e/ou neonatal congênita na Maternidade do Centro de Atençáo Integral 
à Saúde da Mulher, objetivou-se avaliar os métodos para diagnóstico, características da gestação, complicaçóes maternas e resultados perinatais em casos de hidrocefalia congênita, correlacionando-os com variáveis gestacionais e do parto. Verificou-se que o diagnóstico ocorreu antes do parto, sendo confirmado através de exame ecográfico, com parto por cesárea na maioria dos casos. A cefalocentese foi realizada em 11 casos e as complicaçóes no parto vaginal foram mais comuns que na cesárea. $\mathrm{O}$ índice de Apgar baixo foi mais reiterado entre os recém-nascidos de parto vaginal. A hidrocefalia congênita esteve ainda conexa a importante morbidade e mortalidade neonatal e perinatal, outras malformaçóes e número muito pequeno de crianças sem sequelas. Os autores chegaram à conclusáo de que a avaliação destes fatores é importante para que o obstetra acompanhe as gestantes com esta malformação fetal, propiciando desta forma respaldo às decisóes médicas e éticas, e possíveis medidas a serem tomadas [25].

Pesquisa qualitativa, com abordagem sócio-histórica, realizada com profissionais de saúde do Hospital Infantil Joana de Gusmáo que participaram da implantação da UTIN. O objetivo foi compreender como se instituíram as práticas de cuidado ao recém-nascido na Unidade de Terapia Intensiva Neonatal (UTIN) em Florianópolis, na década de 1980. Os autores observaram que através da implantação de uma unidade específica para o cuidado do recém-nascido diminuíra a morbimortalidade neonatal e ainda propiciara aquisição do conhecimento científico. Todos os profissionais de saúde têm o discernimento que, desde os primórdios da UTIN, já havia uma inquietação com o cuidado sensível, humanizado e individualizado ao recém-nascido [26].

Estudo descritivo e exploratório, com abordagem quantitativa, realizado em Fortaleza-CE, teve como sujeitos 24 enfermeiras de três hospitais de referência que foram entrevistadas, utilizando-se um formulário com perguntas abertas e fechadas, de maio a junho/2008. Objetivou-se conhecer a percepção das enfermeiras quanto à influência do desempenho na assistência de enfermagem e no ambiente ocupacional. Os autores chegaram ao entendimento de que concretização da assistência pode ser dificultada pela falta de recursos, sejam materiais e/ou humanos, falta de sensibilização e instrumentalização técnica dos profissionais de saúde, para atendimento das novas necessida- des de trabalho. Desta forma, nas unidades, as atividades são claras, sendo essencial uma equipe unida, harmoniosa e comprometida com assistência de qualidade, na busca da comunicação efetiva, amizade e respeito mútuo [27]. Estudo qualitativo na perspectiva da fenomenologia social teve como objetivo compreender a experiência da enfermeira que atua em unidade de terapia intensiva neonatal. Os autores verificaram que a sobrecarga de atividades, o reduzido número de pessoal, a falta de materiais, equipamentos e a necessidade de refinamento profissional são a realidade do trabalho da enfermeira nesse setor. Desta forma, supervisionar os cuidados é o admissível; cuidar inteiramente do recém-nascido, abrangendo seus pais, é o ideal almejado [28].

\section{Conclusão}

Mediante a análise do estudo sobre as contribuições de enfermagem visando à assistência humanizada ao $\mathrm{RN}$ portador de hidrocefalia e seus familiares, podemos concluir que a enfermagem contribui de maneira efetiva na implementação do cuidado humanizado, porém precisamos de mais discussóes que nos ajudem a fomentar e balizar o direcionamento do caminho que deveremos tomar para que esta prática seja efetuada em todas as unidades assistenciais sejam elas públicas ou privadas. Entendendo que a prática profissional associada às reflexôes do campo prático do fazer e do ser poderá ser a saída de que tanto almejamos, para que a assistência humanizada possa chegar a todos sem distinçáo de raça, cor ou razáo social.

A interação profissional, paciente e seus familiares, pontos importantes neste processo, tem como objetivo maior proporcionar a todos um ambiente livre de negligências, imperícias e imprudências relacionadas ao cuidado. Uma vez que aprendemos serem estes pontos chave para a resolução dos problemas encontrados hoje na prática assistencial, acreditamos que, enquanto categoria de enfermagem, conseguiremos chegar a excelência do cuidado sistematizado, individualizado com base nas solicitaçóes do Programa de Humanização do Sistema Único de Saúde.

\section{Referências}

1. Carvalho V. Dos valores ético-profissionais da Enfermagem contemporânea: consideraçóes filosóficas. Esc Anna Nery Rev Enferm 1997;1(1):33-47. 
2. Carvalho V, Paim L. Acerca da assistência de enfermagem: considerando significado e destaques. Esc Anna Nery Rev Enferm 1999;3(3):37-51.

3. Silva GB. Assistência de enfermagem a um paciente com hidrocefalia internado na clínica pediátrica de um hospital de médio porte do município de Parnaíba-PI. $61^{\circ}$. Congresso Brasileiro de Enfermagem, Fortaleza, Ceará; 2009.

4. Santinho A, Gonçalves S. Refletir para cuidar do paciente com hidrocefalia numa unidade de cuidados de neurocirurgia. Revista Sinais Vitais 2006;65.

5. Marcondes E. Pediatria Básica. 7a . ed. São Paulo: Sarvier; 2005.

6. Kenner C. Enfermagem Neonatal. 2a ed. Rio de Janeiro: Reichmann \& Affonso Editores; 2001.

7. Figueiredo NMA, Viana DL, Machado, Wiliam C. A. Tratado prático de enfermagem $2^{\mathrm{a}}$ ed. Sáo Paulo: Yendis; 2009.

8. Rezende J, Montenegro CAB. Obstetrícia fundamental. $11^{\mathrm{a}}$ ed. Rio de Janeiro: Guanabara Koogan; 2011.

9. Meeker MH, Rothrock JC. Cuidados de enfermagem ao paciente cirúrgico. 10 ed. Rio de Janeiro: Guanabara Koogan; 2007.

10. Alfaro-Lefevre R. Aplicação do processo de enfermagem: um guia passo a passo. Porto Alegre: Artmed; 2000.

11. Carraro TE, Kletemberg DF, Gonçalvez DF. O ensino da metodologia da assistência de enfermagem no Paraná. Rev Bras Enferm 2003;56(5):499-501.

12. Figueiredo NMA. Método e Metodologia na Pesquisa Científica. São Paulo: Difusão; 2004.

13. Andrade MM. Introduçáo à metodologia do trabalho científico. $2^{a}$ ed. São Paulo: Atlas; 1997.

14. Junior RP, Nóbrega SP, Cecatti JG, Barini R, et al. Diagnósticos, conduta obstétrica e resultados perinatais em fetos com hidrocefalia. Rev Bras Ginecol Obstet 1998;20(7);381-7.

15. Kliemann S, Rosemberg S. Hidrocefalia derivada na infância: Um estudo clínico-epidemiológico de 243 observaçóes consecutivas. Arq Neuropsiquiatr 2005;63(2-B): 494-50.

16. Mogrovejo EE, Reto PP, Bazan Q, Cabrera BQ. Complicaciones en el tratamiento quirúrgico de pacientes pediátricos con hidrocefalia operados en el Hospital Nacional Guillermo Almenara Irigoyen. Acta Med Per 2011;28(1):6-11.
17. Lima MMM, Pereira CU, Silva AM. Infecçôes em dispositivos neurológicos implantáveis em crianças e adolescentes. Arq Neuropsiquiatr 2007;65(1):118-23.

18. Oliveira DMP, Pereira CU, Freitas ZMP. Conhecimento do cuidador de crianças com hidrocefalia. Rev Bras Enferm 2010;63(5):782-5.

19. Perlin DA, Oliveira SM, Gomes GC. A criança na unidade de terapia intensiva neonatal: impacto da primeira visita da mãe. Rev Gaúcha Enferm 2011;32(3):458-64.

20. Merighi MAB, Jesus MCP, Santin KR, Oliveira DM. Cuidar do recém-nascido na presença de seus pais: vivência de enfermeiras em unidade de cuidado intensivo neonatal. Rev Latinoam Enferm 2011;19(6):13981404.

21. Souza KMO, Ferreira SD. Assistência humanizada em UTI neonatal: os sentidos e as limitaçóes identificadas pelos profissionais de saúde. Ciênc Saúde Coletiva 2010;15(2):471-80.

22. Sá Neto JA. Enfermagem cuidando do recém-nascido na Unidade de Terapia intensiva Neonatal: um olhar ético da ação profissional [Dissertação]. Rio de Janeiro: Universidade Estadual do Rio de Janeiro; 2009.

23. Klock P, Erdmann AL. Cuidando do recém-nascido em UTIN: convivendo com a fragilidade do viver/ sobreviver à luz da complexidade. Rev Esc Enferm USP 2012;46(1):45-51.

24. Perlin DA, Oliveira SM, Gomes GC. A criança na unidade de terapia intensiva neonatal: impacto da primeira visita da mãe. Rev Gaúcha Enferm 2011;32(3):458-64.

25. Júnior RP, Nóbrega SP, Cecatti JG, Barini R. Diagnóstico, conduta obstétrica e resultados perinatais em fetos com hidrocefalia. Rev Bras Ginecol Obstet 1998;20(7):381-7.

26. Costa R, Padilha MI. A Unidade de Terapia Intensiva Neonatal possibilitando novas práticas no cuidado ao recém-nascido Rev Gaúcha Enferm 2011;32(2):24855.

27. Cardoso SNM, Esteche CMGE, Oliveira MMC, Sherlock MSMO. Desafios e estratégias das enfermeiras na unidade de terapia intensiva Neonatal. Rev Rene 2010;11(4):76-84.

28. Montanholi LL, Merighi MAB, Jesus MCP. Atuação da enfermeira na unidade de terapia intensiva neonatal: entre o ideal, o real e o possível. Rev Latinoam Enferm 2011;19(2):301-8. 